\title{
Plant-Microbe Communication Enhances Auxin Biosynthesis by a Root-Associated Bacterium, Bacillus amyloliquefaciens SQR9
}

\author{
Yunpeng Liu, ${ }^{1}$ Lin Chen, ${ }^{2}$ Nan Zhang, ${ }^{2}$ Zunfeng Li, ${ }^{2}$ Guishan Zhang, ${ }^{1} \mathrm{Yu} \mathrm{Xu},{ }^{2}$ Qirong Shen, ${ }^{2}$ and \\ Ruifu Zhang ${ }^{1,2}$ \\ ${ }^{1}$ Key Laboratory of Microbial Resources Collection and Preservation, Ministry of Agriculture, Institute of Agricultural Resources \\ and Regional Planning, Chinese Academy of Agricultural Sciences, Beijing 100081, P.R. China; and ${ }^{2}$ Jiangsu Key Lab and \\ Engineering Center for Solid Organic Waste Utilization, National Engineering Research Center for Organic-based Fertilizers, \\ Nanjing Agricultural University, Nanjing, 210095, P.R. China
}

Submitted 20 October 2015. Accepted 15 January 2016.

\begin{abstract}
Mechanisms by which beneficial rhizobacteria promote plant growth include tryptophan-dependent indole-3-acetic acid (IAA) synthesis. The abundance of tryptophan in the rhizosphere, however, may influence the level of benefit provided by IAAproducing rhizobacteria. This study examined the cucumberBacillus amyloliquefaciens SQR9 system and found that SQR9, a bacterium previously shown to enhance the growth of cucumber, increased root secretion of tryptophan by three- to fourfold. Using a split-root system, SQR9 colonization of roots in one chamber not only increased tryptophan secretion from the noninoculated roots but also increased the expression of the cucumber tryptophan transport gene but not the anthranilate synthesis gene in those roots. The increased tryptophan in isolated rhizosphere exudates was sufficient to support increased IAA production by SQR9. Moreover, SQR9 colonization of roots in one chamber in the split-root system resulted in sufficient tryptophan production by the other roots to upregulate $S Q R 9$ IAA biosynthesis genes, including a 27-fold increase in the indole-3-acetonitrilase gene $\boldsymbol{y h c X}$ during subsequent colonization of those roots. Deletion of $y \boldsymbol{h c X}$ eliminated SQR9-mediated increases in root surface area, likely by reducing IAA-stimulated lateral root growth. This study demonstrates a chemical dialogue between $B$. amyloliquefaciens and cucumber in which this communication contributes to bacteria-mediated plantgrowth enhancement.
\end{abstract}

Many root-associated bacteria can increase crop yield, and they provide significant advantages over chemical fertilizers for reducing environmental pollution (Germida and Walley 1996; Shaharoona et al. 2008). Consequently, many researchers have investigated the interactions between these bacteria and plants (Germida and Walley 1996; Shaharoona et al. 2008; Zhang et al. 2014).

Some bacteria directly benefit plants via the synthesis and secretion of a range of phytohormones, including indole-3-acetic acid (IAA). IAA production is one of the main mechanisms involved in plant-growth promotion by rhizobacteria. Its production

Corresponding author: R. Zhang; E-mail: zhangruifu@caas.cn; Telephone: 86-10-82108634; Fax: 86-10-82108683.

*The $\boldsymbol{e}$-Xtra logo stands for "electronic extra" and indicates that two supplementary figures and one supplementary table are published online.

(c) 2016 The American Phytopathological Society by root-associated bacteria can lead to stimulation of root production and, consequently, plant growth (Bloemberg and Lugtenberg 2001).

Several IAA synthesis pathways in plants and microbes have been characterized, and some key genes and enzymes that participate in these pathways have been identified. Tryptophan is a major substrate for IAA synthesis in rhizobacteria (Hull et al. 2000; Idris et al. 2007; Shao et al. 2015a and b; Wright et al. 1991). In general, the tryptophan-dependent IAA synthesis pathways include the indole-3-acetamide, indole-3-pyruvate, tryptamine, and indole-3-acetonitrile pathways (Spaepen et al. 2007). The biosynthesis of IAA by bacteria living in the rhizosphere is affected by root exudates (Bais et al. 2006), and the composition of root exudates depends on factors such as the plant species and microbial colonization of the rhizosphere (Bais et al. 2008). To date, studies of the mechanisms by which rhizobacteria promote plant growth have mainly focused on the unidirectional contribution of rhizobacteria to the plant. However, the effect of plantmicrobe communication on bacteria-stimulated growth promotion is poorly understood.

In this study, we demonstrated that plant-microbe communication can contribute to auxin synthesis by the beneficial root-associated strain Bacillus amyloliquefaciens SQR9 and to plant-growth promotion. Cucumber secreted more tryptophan in root exudates upon colonization by Bacillus amyloliquefaciens SQR9 and, in turn, SQR9 synthesized more IAA in the rhizosphere, using the root-secreted tryptophan to promote plant growth.

\section{RESULTS}

Colonization of cucumber roots by $B$. amyloliquefaciens SQR9 enhanced the secretion of tryptophan.

To test the effect of rhizobacteria on root exudates, a split-root system was applied that divided cucumber roots into two parts, each of which was located in a distinct chamber of the root box (Fig. 1). The roots in the left chamber were either inoculated with bacteria or were not inoculated as a control, while the right chamber was used to collect the root exudates. In this manner, the impact of the bacteria on the plant production of exudates could be separated from the impact of the bacteria on the root exudates themselves, such as via catabolism. The root exudates collected from SQR9-colonized and noncolonized split-root systems (Fig. 1A) were analyzed by high-performance liquid chromatography (HPLC), and the results showed that 
SQR9-colonized cucumber roots secreted threefold more tryptophan than the control cucumber roots (Fig. 2A and B). To test the effect of other rhizosphere microbes on cucumber root tryptophan secretion, plants were treated in the same manner as with SQR9 but with the cucumber wilt pathogen Fusarium oxysporum f. sp. cucumerinum, a beneficial Pseudomonas brassicacearum J12 strain (Zhou et al. 2012), and Bacillus subtilis 168 , which is not known to benefit plants. None of these microbes enhanced the secretion of tryptophan by cucumber roots (Fig. 2A). The high-throughput gas chromatography mass spectrometry
(GC-MS) analysis of the cucumber root exudates yielded similar results (Fig. 2C); the SQR9-colonized cucumber root exudates produced approximately four times more tryptophan based on peak height than the control root exudates.

The transcription of a cucumber tryptophan transport gene but not a synthesis gene was upregulated upon B. amyloliquefaciens SQR9 colonization.

To verify the above observation and explore the mechanism responsible for the enhanced tryptophan secretion, the responses

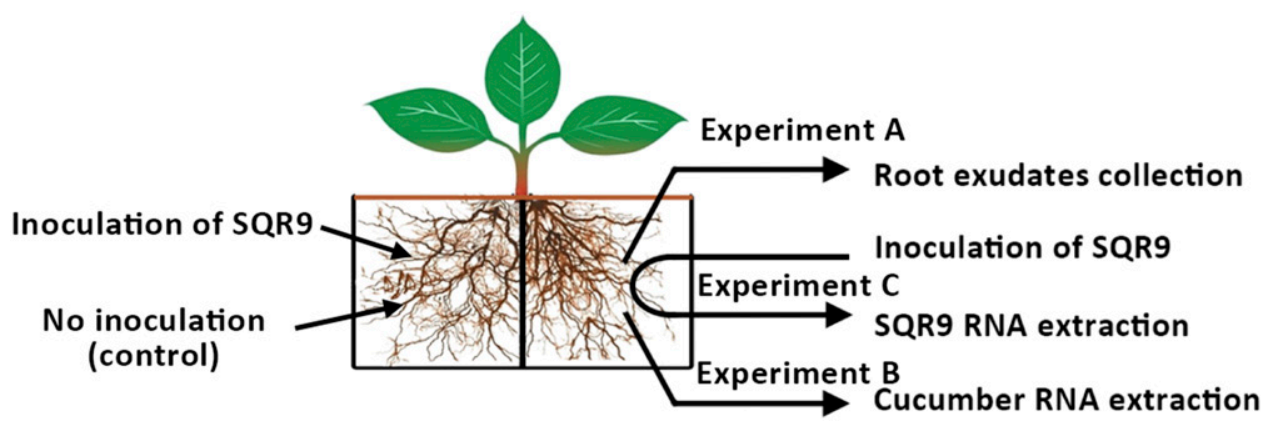

Fig. 1. Split-root experimental design. Experiment A involved root exudate collection. In the split-root system, the left chamber was inoculated with Bacillus amyloliquefaciens SQR9, Pseudomonas brassicacearum J12, the pathogen Fusarium oxysporum f. sp. cucumerinum, or Bacillus subtilis 168 or was not inoculated, as a control. The root exudates were collected from the right chamber 2 days later. Three replicates were included for each treatment. In experiment B, the left chamber was either inoculated with Bacillus amyloliquefaciens SQR9 or was not inoculated, as a control. Two days after inoculation of the left side by SQR9, the RNA from roots in the right chamber were extracted and the transcription of cucumber genes was evaluated. Three biological replicates were included for each treatment. In experiment C, the left chamber was either inoculated with Bacillus amyloliquefaciens SQR9 or was not inoculated, as a control. Two days later, the right chamber was inoculated with B. amyloliquefaciens SQR9, which was allowed to colonize the roots. RNA was extracted from the SQR9 cells in the right chamber $48 \mathrm{~h}$ after inoculation to further evaluate the transcription of genes related to indole 3-acetic acid synthesis. Three replicates were included for each treatment.

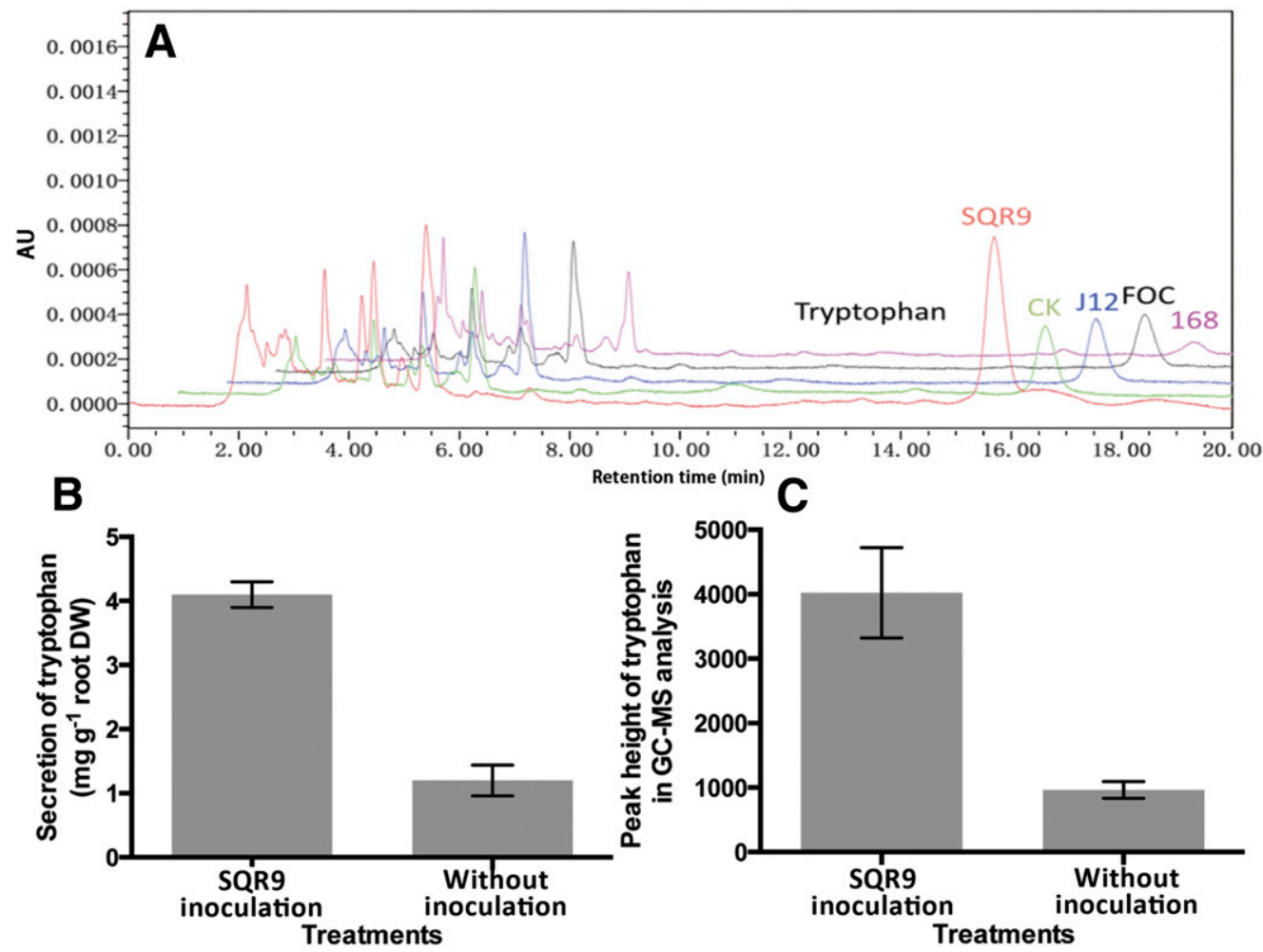

Fig. 2. Tryptophan detection in root exudates. Root exudates were collected at 2 days after inoculation and were freeze-dried and powdered for analysis A, High-performance liquid chromatography spectrum. The root exudates of cucumbers colonized by Bacillus amyloliquefaciens SQR9 contained significantly more tryptophan than the exudates of noncolonized roots (CK). Inoculation with Pseudomonas brassicacearum J12, the pathogen Fusarium oxysporum f. sp. cucumerinum (FOC), and the strain Bacillus subtilis 168 did not significantly affect the tryptophan content in root exudates. The tryptophan peaks are shown on the right and are offset for clarity. B, Quantification of tryptophan in root exudates based on a standard curve. C, Relative quantification by gas chromatography mass spectrometry. For each column, error bars indicate the standard deviations based on three repeated experiments. 
to SQR9 root colonization of two cucumber genes related to tryptophan synthesis and transport were investigated (Fig. 1B). This was similarly performed using a split-root system in which the RNA was collected from roots in a separate chamber from the SQR9-inoculated roots. The results showed that the transcription of a tryptophan transporter gene (Csa024547), which is the only specific tryptophan transporter gene in the cucumber genome, continued to increase during the two days following SQR9 colonization and, ultimately, reached a level 40-fold higher than that of cucumber plants not inoculated with SQR9. However, the gene encoding anthranilate synthase (Csa013682) did not exhibit significant differences in transcription (Fig. 3).

\section{B. amyloliquefaciens SQR9 synthesized IAA} using root-secreted tryptophan in the rhizosphere.

To investigate whether the increased tryptophan content in root exudates would affect the ability of SQR9 to synthesize IAA, the IAA levels produced by SQR9 in mineral medium supplied with the following amendments were compared: no amendment, $8 \mathrm{mg}$ tryptophan per liter, cucumber root exudates from uninoculated plants, cucumber root exudates from plants inoculated with SQR9 in the split-root system, and cucumber root exudates from uninoculated plants but amended with $8 \mathrm{mg}$ of tryptophan per liter. The results presented in Figure 4 showed that, compared with the unamended cells and cucumber root exudates from uninoculated plants, SQR9 exhibited significantly increased IAA production in cultures amended with cucumber root exudates from SQR9-colonized plants, tryptophan, and cucumber root exudates from uninoculated plants plus extra tryptophan.

\section{B. amyloliquefaciens SQR9 enhanced plant growth} in response to increased tryptophan in the rhizosphere.

To verify that the induced tryptophan secretion can further improve the ability of SQR9 to promote plant growth in the rhizosphere, the growth of cucumber plants in a gnotobiotic system with SQR9 was evaluated in the presence and absence of exogenous tryptophan. Inoculating plants with SQR9 at a final concentration of $10^{6} \mathrm{CFU} \mathrm{ml^{-1 }}$ together with exogenous tryptophan showed markedly higher cucumber growth compared with SQR9 alone without exogenous tryptophan, as shown by

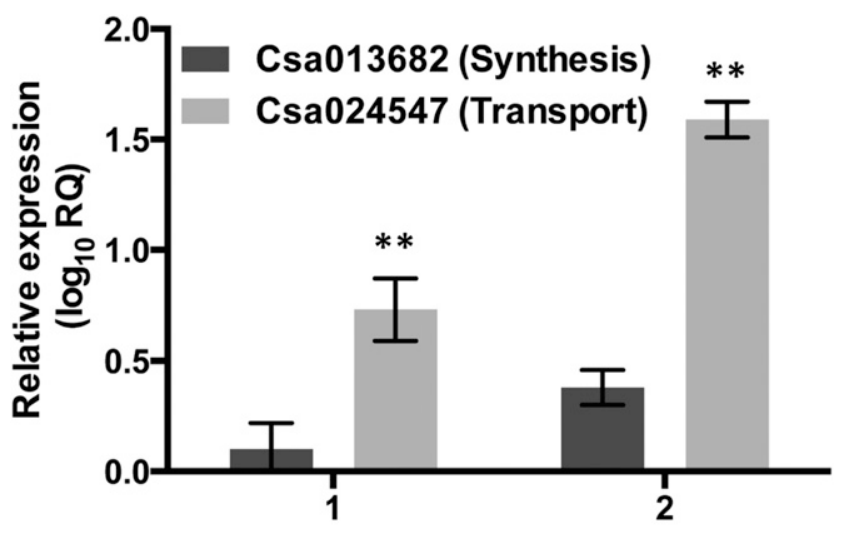

Day after inoculaiton

Fig. 3. Transcription of genes in cucumber. Csa013682 encodes anthranilate synthase, the key enzyme involved in tryptophan synthesis in plants. Csa024547 encodes a tryptophan-specific transporter. Values are relative to the same genes in cucumber without inoculation. The transporter gene was up-regulated upon inoculation with SQR9, whereas the synthesis gene remained unchanged. For each column, error bars indicate the standard deviations, based on three different repeated experiments. Double asterisks above the bars indicate significant differences compared with no inoculation control $(P<0.01)$. cucumber seedling size and biomass (Fig. 5A and B, respectively). The addition of tryptophan in the absence of SQR9 inoculation did not affect cucumber growth, and inoculating roots with $10^{6} \mathrm{CFU}$ of SQR9 per milliliter plus tryptophan resulted in unusual root architecture and a twofold increase in total root-surface area (Fig. 5C). However, tryptophan did not significantly affect the root dry weight (Supplementary Fig. S1).

An SQR9 $y h c X$ gene deletion mutant, which exhibits a $24 \%$ reduction in tryptophan-dependent IAA production due to loss of a gene in the indole-3-acetonitrile pathway (Shao et al. 2015a), did not enhance plant growth in response to additional tryptophan when inoculated at a density of $10^{6} \mathrm{CFU} \mathrm{ml}^{-1}$ with $8 \mathrm{mg}$ of tryptophan per liter (Fig. 6).

\section{Transcription of IAA synthesis genes was upregulated in root-colonized $B$. amyloliquefaciens SQR9.}

To investigate the transcriptional response of SQR9 to root colonization following SQR9-mediated induction of enhanced tryptophan secretion, SQR9 was either preinoculated into the left chamber of a split-root system or not, as a control, and 2 days later, a subsequent inoculation of SQR9 was performed in the right chamber (Fig. 1C). The plant was then allowed to grow for another 2 days before RNA extraction using a bacterial RNA extraction kit. The quantitative polymerase chain reaction (qPCR) results showed that the tryptophan-dependent IAA synthesis genes were significantly upregulated in roots colonized with SQR9 when another part of the root was precolonized with SQR9, compared with the control treatment (Fig. 7). The transcription of the gene $y h c X$ was up-regulated 27-fold (Fig. 7). This induction is consistent with the accumulation of tryptophan in root exudates induced by preinoculation with SQR9.

\section{DISCUSSION}

Our previous study showed that tryptophan-dependent IAA synthesis is a major mechanism by which $B$. amyloliquefaciens SQR9 promotes plant growth (Shao et al. 2015a and b). The critical finding demonstrated in this study is that the colonization of cucumber roots by $B$. amyloliquefaciens SQR9 stimulates the transcription of the root tryptophan transporter

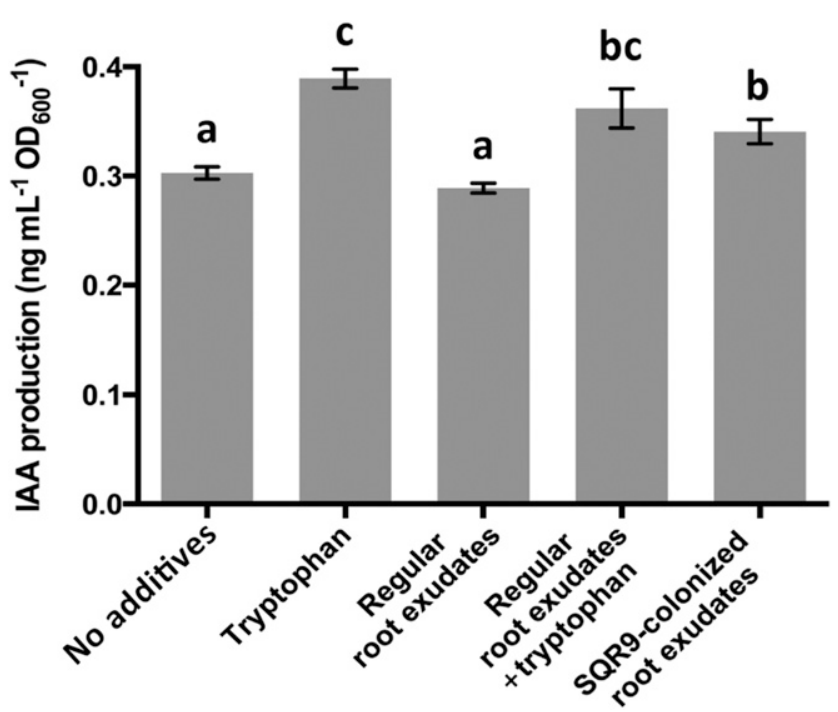

Fig. 4. Indole 3-acetic acid (IAA) production of SQR9 in response to root exudates and tryptophan. The results were normalized to the concentration of cells. Error bars indicate the standard deviations based on three repeated experiments. Different letters above the bars indicate significant differences $(P<0.05)$. 
gene and, consequently, enhances tryptophan secretion. SQR9 subsequently uses this tryptophan as a substrate for IAA synthesis, which, in turn, benefits plant growth. Thus, the collective results support the model that the plant-growth promotion in rhizosphere was affected by altered root exudation induced by SQR9 inoculation (Fig. 8).

At present, factors regulating the expression of plantbeneficial traits are poorly understood. Root-secreted tryptophan is known to be important to the ability of many beneficial bacteria that promote plant growth. However, past studies have not considered the source of the tryptophan and, in particular, whether the root exudate composition is a variable factor that is influenced by plant-microbe communication (Zhang et al. 2014). As reported, the tryptophan content in cucumber root exudates is not sufficient to support detectable IAA production by bacteria (Kamilova et al. 2006a and b). Our demonstration that cucumber roots increase tryptophan secretion in response to colonization by Bacillus amyloliquefaciens SQR9, increasing subsequent IAA production by SQR9, illustrates a bidirectional communication process between this rhizosphere bacterium and the plant. In combination with our data showing increased plant growth by SQR9 (Shao et al. 2015a and b) (Fig. 5), these findings suggest that plant-microbe communication can be an important component of bacterial plant-growth promotion.

An interesting question is whether this cross-talk leading to plant-growth promotion is common for microbes and plants. In this study, a beneficial bacterium from the tomato rhizosphere, Pseudomonas brassicacearum J12 (Zhou et al. 2012), failed to induce the secretion of tryptophan from cucumber roots. Similarly, the beneficial bacterium Pseudomonas fluorescens WCS365 from the potato rhizosphere did not increase the growth of cucumber that secreted a low amount of tryptophan in root exudates, but it did increase the growth of other plants with higher amounts of tryptophan in root exudates (Geels and Schippers 1983, Kamilova et al. 2006a and b). The fact that SQR9 was originally isolated from the cucumber rhizosphere may suggest that the specificity of this SQR9-cucumber crosstalk is a reflection of coevolution of the plant host and rhizobacterium.

\section{MATERIALS AND METHODS}

Growth condition of strain and plant.

Bacillus amyloliquefaciens SQR9 (China General Microbiology Culture Collection Center accession number 5808), Pseudomonas brassicacearum J12 (National Center for Biotechnology Information accession number: JN605747), and Bacillus subtilis 168 (Bacillus Genetic Stock Center) were grown at $37^{\circ} \mathrm{C}$ in Luria-Bertani medium (LB). The $\Delta y h c X$ mutant strain of SQR9 (Shao et al. 2015a) was grown at $37^{\circ} \mathrm{C}$ in LB medium containing $4 \mu \mathrm{g}$ of chloramphenicol and $20 \mu \mathrm{g}$ of zeocin per milliliter. The fungal pathogen Fusarium oxysporum f. sp. cucumerinum NJAU-2 was stored at the Jiangsu Provincial Key Laboratory for Organic Solid Waste Utilization, Nanjing Agricultural University and was maintained on potato

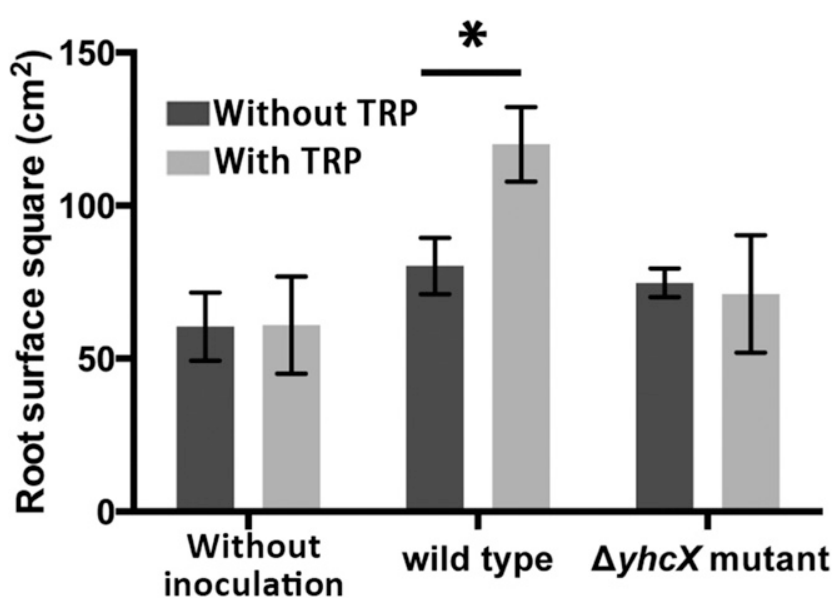

Fig. 6. Verification of effect of the $y h c X$ mutant on the root surface area. Plants were inoculated with $10^{6}$ cells of SQR9 (wild type) and $\Delta y h c X$, both with and without tryptophan added at a concentration of $8 \mathrm{mg}$ per liter. Error bars indicate the standard deviations based on at least five repeated experiments. Asterisks above the bars indicate significant differences $(P<0.05)$.

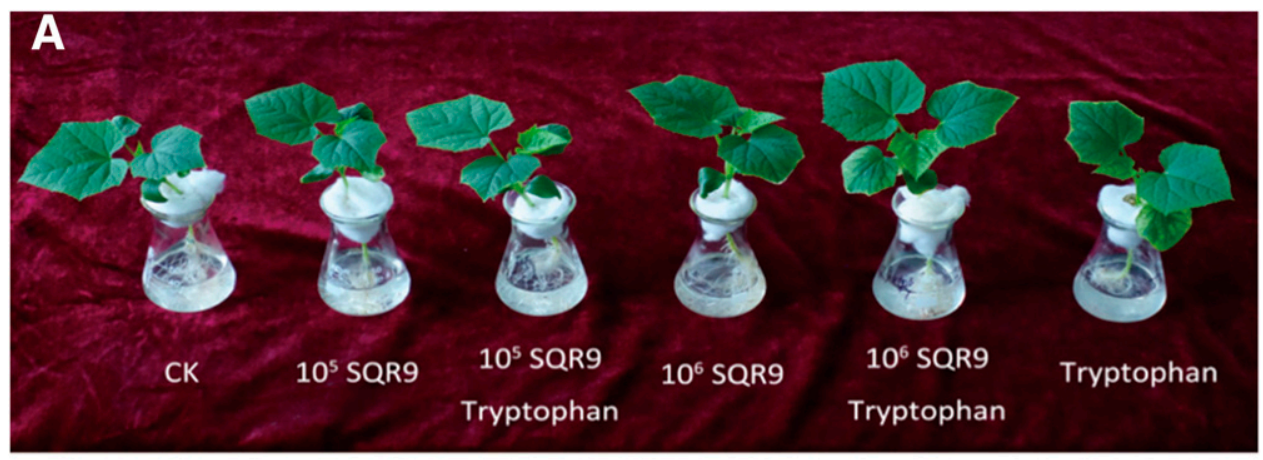

B

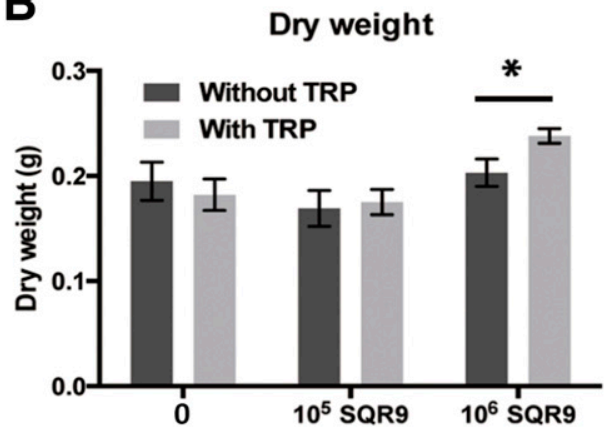

C

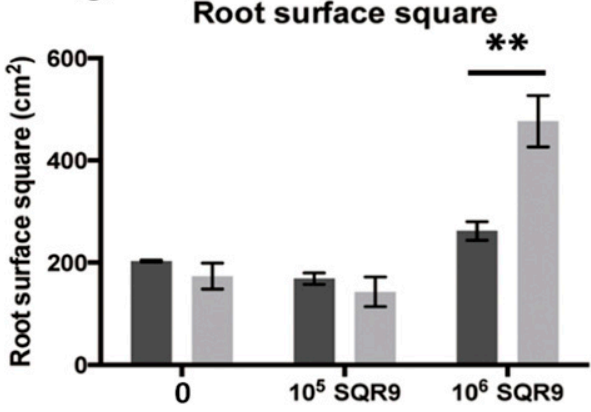

Fig. 5. Growth promotion of SQR9 with exogenous tryptophan. Tryptophan was added at a concentration of $8 \mathrm{mg}$ per liter. A, Plant growth, B, plant dry weight, and $\mathbf{C}$, root surface area. Error bars indicate the standard deviations based on five repeated experiments. Asterisks above the bars indicate significant differences $(P<0.05)$. 
dextrose agar plates. A conidial suspension of Fusarium oxysporum f. sp. cucumerinum was prepared as described previously (Cao et al. 2011).

Cucumber seeds of the cultivar Jinchun 4 were surfacedisinfected in $2 \% \mathrm{NaClO}$ solution for $15 \mathrm{~min}$, were washed thoroughly with distilled water, and were planted in axenic tissue culture bottles containing vermiculite. The seeds were allowed to germinate and grow for approximately 4 days in a growth chamber at $23^{\circ} \mathrm{C}$ with a 16 -h light and 8-h dark photoperiod, and the seedlings were then aseptically and individually transplanted into 50-ml flasks containing $35 \mathrm{ml}$ (to emerge the seedling roots in the medium) of sterile liquid 1/4 sucrose-free Murashige Skoog (MS) medium (Murashige and Skoog 1962). The seedlings were then allowed to grow for another 3 weeks with gentle shaking $(50 \mathrm{rpm})$ for $2 \mathrm{~h}$ per day on a shaker. The



Fig. 7. Gene transcription of Bacillus amyloliquifaciens SQR9 in the rhizosphere. Values indicate the relative transcription of genes related to indole 3-acetic acid (IAA) synthesis by SQR9 on roots precolonized by SQR9 compared with that on roots of cucumber without precolonization. Gene expression was examined after $48 \mathrm{~h}$ of SQR9 colonization on roots in the right chamber. In SQR9, $y c l B C$ is within the same gene cluster as genes encoding an aromatic acid decarboxylase, which catalyzes the decarboxylation of indole-3-pyruvate to indole-3-acetaldehyde. dhaS encodes indole aldehyde dehydrogenase, which catalyzes the conversion of indole3 -acetaldehyde into IAA. $y h c X$ encodes a putative nitrilase involved in the indole acetonitrile pathway. $y b g E$ encodes a branched-chain amino acid aminotransferase involved in the tryptophan side-chain oxidase pathway. Error bars indicate the standard deviations based on three repeated experiments.
MS medium was replaced every other day during the growth period.

For root exudates collection and the colonization assay, the cucumber seedlings were transferred to a sterilized split-root system (six plants in each), which consisted of a glass wall to completely separate the root chamber into two halves (Fig. 1). The seedlings were then cultured for 2 more weeks in 1/4 sucrose-free MS medium. Prior to inoculation with B. amyloliquefaciens SQR9 and other tested strains, a 100- $\mu \mathrm{l}$ aliquot of the root medium was spread on solid LB medium to test for contamination. Contaminated systems were discarded.

\section{Exudates collection and RNA extraction from cucumber roots.}

After 2 weeks of culturing in the split-root system, one chamber of the system was inoculated with SQR9 cell suspensions at a final concentration of $5 \times 10^{6} \mathrm{ml}^{-1}$. Noninoculated chambers served as a control (Fig. 1A). Then, two days later, the medium in the other side chamber was replaced with sterilized double-distilled water and was cultured, at $23^{\circ} \mathrm{C}$ with a 16-h light and 8-h dark photoperiod for 4 days, to collect the exudates from the noninoculated root side. Root exudates were collected every day during the four-day period (Fig. 1A). To investigate the specificity of the effect of SQR9, the plants were treated with Pseudomonas brassicacearum J12, the cucumber soil-borne fungal pathogen $F$. oxysporum $\mathrm{f}$. sp. cucumerinum, and Bacillus subtilis 168, instead of SQR9. All treatments consisted of three replicates (containers), and each container contained six plants. For each of the three replicates, the root exudate solutions collected during the four days were pooled and, then, were filtered through a $0.45-\mu \mathrm{m}$ membrane (Millipore) and the sterility of the filtered exudates was tested by plating $100 \mu \mathrm{l}$ of exudate on $\mathrm{LB}$ plates and incubating the plates at $30^{\circ} \mathrm{C}$ for 3 days. The root exudates were freeze-dried and powdered for further use. The roots from which the exudates were collected were cut, and the concentrations of root exudates were normalized to the dry weights of these roots.

Additionally, two days after inoculation with SQR9, the roots from which the exudates were collected were cut, were flashfrozen in liquid nitrogen, and were ground to a powder for RNA extraction using a Qiagen RNeasy plant mini kit (Fig. 1B). The extracted RNA samples were evaluated on a $1 \%$ agarose gel, and their RNA concentrations and quality (A260/A280) were determined using a NanoDrop ND-2000 spectrophotometer (NanoDrop). RNA was extracted from three plants per treatment.

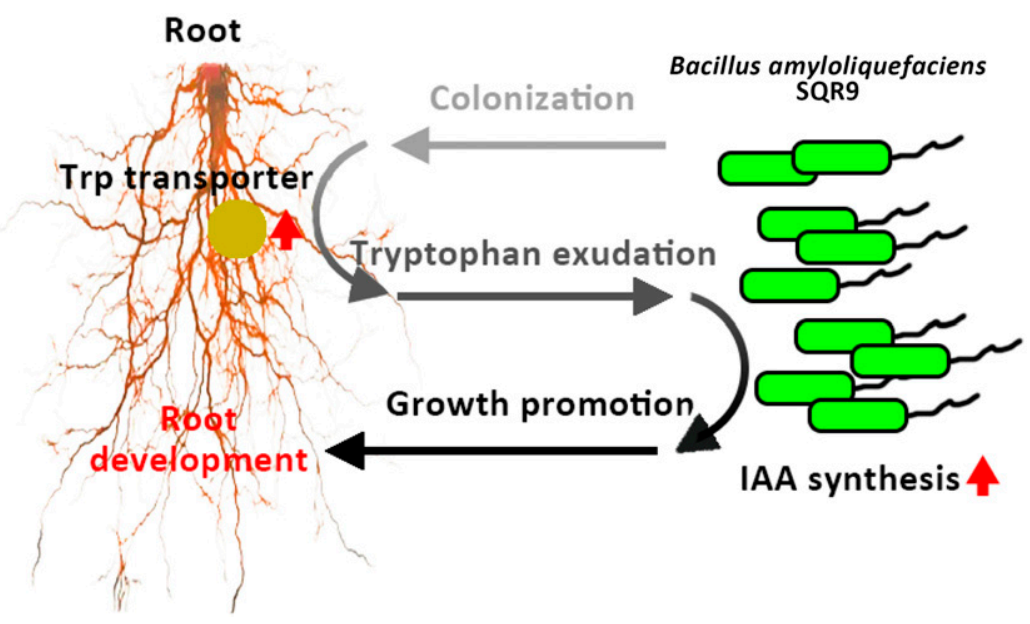

Fig. 8. Model of cucumber-Bacillus amyloliquifaciens interactions. Colonization of plant roots by B. amyloliquefaciens SQR9 upregulates the expression of the tryptophan transporter in cucumber roots, which increases tryptophan secretion by the roots. SQR9 then senses this tryptophan and produces indole 3 -acetic acid (IAA) to consequently benefit plant growth. 


\section{Quantification of tryptophan in root exudates.}

The concentration of tryptophan was measured using two methods, HPLC analysis and GC-MS, which were carried out at Nanjing Agricultural University and the University of California Davis, respectively.

For the HPLC analysis, lyophilized root exudates were dissolved in ultra-pure water. A tryptophan standard of chromatographic grade was purchased from Sigma-Aldrich. Ultra-pure water with a specific resistance of $18.3 \mathrm{M} \Omega$ per centimeter (Milli-Q Plus system, Millipore) was used to prepare all solutions and the tryptophan standard. Ten microliters of sample solution was injected for HPLC analysis. A Waters e2695 Separations module was used to separate the components of solutions, and the $2489 \mathrm{UV} / \mathrm{Vis}$ detector was used to detect the components. The column was an Xbridge-C18 $(5 \mu \mathrm{m}, 4.6 \times 250 \mathrm{~mm})$ and the mobile phase consisted of a 95:5 mixture of $\mathrm{A}$ and $\mathrm{B}$, where A was $\mathrm{pH} 4.0 \mathrm{CH}_{3} \mathrm{COONa}$ solution $\left(\mathrm{c}\left[\mathrm{Na}^{+}\right]=8.5 \mathrm{mM}\right)$ and $\mathrm{B}$ was methanol. The temperature of the column was set at $25^{\circ} \mathrm{C}$, and the detector wavelength was set to $280 \mathrm{~nm}$. A standard curve was generated using a tryptophan gradient. The final concentration was calculated based on peak area and was then normalized to the root dry weight and number of collecting days.

For the GC-MS analysis, freeze-dried root exudates were shipped to the Genome Center Core at the University of California Davis for analyses. The sample program and analysis were performed as described (Badri et al. 2013).

\section{Plant-growth promotion assay.}

Bacillus amyloliquefaciens SQR9 and its $\Delta y h c X$ mutant strain were washed with sterilized water and were suspended in $1 / 4$ sucrose-free MS medium (optical density at $600 \mathrm{~nm}\left[\mathrm{OD}_{600}\right]=$ 0.6). The effect of exogenous tryptophan and SQR9 on the growth of cucumber plants was assessed using the following six treatments: i) a control treatment, consisting of plants without tryptophan or SQR9, ii) adding tryptophan only, iii) adding $10^{5}$ CFU of SQR9 per milliliter, iv) adding $10^{5}$ CFU of SQR9 and tryptophan per milliliter, v) adding $10^{6} \mathrm{CFU}$ of SQR9 per milliliter, and vi) adding $10^{6} \mathrm{CFU}$ of SQR9 and tryptophan per milliliter. A subsequent experiment for comparing the effect of SQR9 $\Delta y h c X$ mutant was subsequently tested as follows: i) control treatment, consisting of plants without tryptophan or SQR9, ii) tryptophan-only treatment, iii) treatment with $10^{6}$ of SQR9 per milliliter, iv) treatment with $10^{6}$ of SQR9 and tryptophan per milliliter, v) treatment with $10^{6}$ of $\Delta y h c X$ mutant per milliliter, and vi) treatment with $10^{6}$ of $\Delta y h c X$ mutant and tryptophan per milliliter. Based on the analysis of tryptophan in the root exudates, tryptophan was added to a final concentration of $8 \mathrm{mg}$ per liter for all treatments that included tryptophan. The underground part of the system was covered with black to avoid the effect of light on IAA. After treatment for one week, the dry weight and root index of each plant was measured to evaluate plant growth. All treatments and controls were repeated five times.

\section{SQR9 IAA production} with different cucumber root exudates.

A culture experiment was performed to evaluate the IAA production by $B$. amyloliquefaciens $\mathrm{SQR} 9$ under different conditions. B. amyloliquefaciens SQR9 was grown at $37^{\circ} \mathrm{C}$ in $\mathrm{LB}$ medium for $8 \mathrm{~h}$, and $1 \mathrm{ml}$ of the culture was centrifuged and washed with sterile water. The cells were resuspended and cultured in mineral medium with glucose (per liter, $20 \mathrm{~g}$ of glucose, $2.0 \mathrm{~g}$ of $\left(\mathrm{NH}_{4}\right)_{2} \mathrm{SO}_{4}, 0.2 \mathrm{~g}$ of $\mathrm{MgSO}_{4} \cdot 7 \mathrm{H}_{2} \mathrm{O}, 0.01 \mathrm{~g}$ of $\mathrm{CaCl}_{2} \cdot 2 \mathrm{H}_{2} \mathrm{O}, 0.001 \mathrm{~g}$ of $\mathrm{FeSO}_{4} \cdot 7 \mathrm{H}_{2} \mathrm{O}, 1.5 \mathrm{~g}$ of $\mathrm{Na}_{2} \mathrm{HPO}_{4} \cdot 12 \mathrm{H}_{2} \mathrm{O}$, and $1.5 \mathrm{~g}$ of $\mathrm{KH}_{2} \mathrm{PO}_{4}$ ) at $25^{\circ} \mathrm{C}$ and $140 \mathrm{rpm}$ in the dark for $48 \mathrm{~h}$, and different substrates were then added to the culture medium as follows: i) $8 \mathrm{mg}$ of tryptophan per liter, ii) regular cucumber root exudate, iii) regular cucumber root exudate plus $8 \mathrm{mg}$ of tryptophan per liter, and iv) SQR9-colonized cucumber root exudates. The concentrations of the root exudates in these treatments were the same as those in the exudates collected from hydroponic plants. To monitor the growth curves of SQR9 for these treatments, cells were harvested $6,8,10,12,14,16$, $18,20,22,24,26,28$, and $34 \mathrm{~h}$ after inoculation, and their $\mathrm{OD}_{600}$ was measured (Supplementary Fig. S2). The supernatant collected at $48 \mathrm{~h}$ was also concentrated to detect IAA with an enzyme-linked immunosorbent assay (ELISA) using an IAA ELISA kit (Shanghai Lengton Bioscience Co., Ltd.). Finally, IAA production was normalized to the cell number.

\section{RNA extraction of SQR9 that colonized on cucumber roots.}

After transplanting the cucumber seedlings from the hydroponic flask to the split-root system and allowing them to grow for 2 weeks, one chamber was inoculated with a final concentration of $5 \times 10^{6}$ of SQR9 per milliliter; noninoculated chambers served as a control. Two days later, suspensions of SQR9 were added to the other chambers of both the SQR9 precolonized treatment and the control experiment (Fig. 1), and the seedlings were allowed to grow for an additional two days. The roots that received a secondary inoculation were cut and flash-frozen in liquid nitrogen for RNA extraction using the e.Z.N.A bacterial RNA kit (OMEGA Bio-tek) (Fig. 1C). The extracted RNA samples were evaluated on a $1 \%$ agarose gel, and the RNA concentration and quality (A260/A280) were determined using a NanoDrop ND-2000 spectrophotometer (NanoDrop). Each sample consisted of three replicates.

\section{Gene transcription quantification.}

Reverse-transcription PCR was performed for RNA extracted from both cucumber plants and SQR9, using the PrimeScript RT reagent kit (Takara). The concentration of cDNA was measured using a NanoDrop ND-2000 spectrophotometer (NanoDrop), and RNA samples were diluted as necessary for real-time qPCR, which was used to quantify the transcription of genes related to IAA synthesis in B. amyloliquefaciens SQR9 and genes related to tryptophan synthesis and transport in cucumber plants. Real time qPCR was performed using an ABI 7500 Cycler (Applied Biosystems). The 20- $\mu$ l reaction solution consisted of $10 \mu \mathrm{l}$ of SYBR Premix EX Taq (2×; TaKaRa), $0.4 \mu$ l of each primer $(10 \mu \mathrm{M}), 0.4 \mu \mathrm{l}$ of ROX reference dye II (50x), $2 \mu \mathrm{l}$ of template DNA, and $6.8 \mu \mathrm{l}$ of double distilled $\mathrm{H}_{2} \mathrm{O}$. The thermal cycling conditions were set to the following: $30 \mathrm{~s}$ at $95^{\circ} \mathrm{C}$ for initial denaturation, followed by 40 cycles of $5 \mathrm{~s}$ at $95^{\circ} \mathrm{C}$ and $34 \mathrm{~s}$ at $60^{\circ} \mathrm{C}$. The two target genes in cucumber plants, Csa013682, which encodes anthranilate synthase, and Csa024547, which encodes a tryptophan transporter (Cucurbit Genomics database), were quantified using an ubiquitin extension proteincoding gene as an internal reference (Huang et al. 2009; Wan et al. 2010); the primers are shown in Supplementary Table S1.

The transcription levels of six genes of SQR9 involved in IAA synthesis, including $y h c X, y c l C, y c l B, y h d R, y b g E$, and $d h a S$ (Shao et al. 2015a), were measured using recA as an internal reference. The specificity of the amplification was verified by a melting-curve analysis and agarose gel electrophoresis. The relative transcription levels were calculated using the $2^{-\Delta \Delta C T}$ method, as reported previously (Livak and Schmittgen 2001).

\section{Statistical analysis.}

Differences among the multiple treatments were calculated and statistically analyzed using an analysis of variance and Duncan's multiple range tests $(P<0.05)$. Pairwise comparisons 
were performed using Student's $t$ test. SPSS version 20.0 was used for the statistical analysis (SPSS Inc.).

\section{ACKNOWLEDGMENTS}

This research was financially supported by National Natural Science Foundation of China (41271271 and 31330069), the National Key Basic Research Program of China (973 program, 2015CB150505) and the National Infrastructure of Microbial Resources (NIRM). R. Zhang and Q. Shen were also supported by the 111 Project (B12009) and the Priority Academic Program Development (PAPD) of Jiangsu Higher Education Institutions.

\section{LITERATURE CITED}

Badri, D. V., Chaparro, J. M., Zhang, R., Shen, Q., and Vivanco, J. M. 2013. Application of natural blends of phytochemicals derived from the root exudates of Arabidopsis to the soil reveal that phenolic-related compounds predominantly modulate the soil microbiome. J. Biol. Chem. 288:4502-4512.

Bais, H. P., Broeckling, C. D., and Vivanco, J. M. 2008. Root exudates modulate plant-Microbe interactions in the rhizosphere. Pages 241-252 in: Secondary Metabolites in Soil Ecology. Springer, Berlin.

Bais, H. P., Weir, T. L., Perry, L. G., Gilroy, S., and Vivanco, J. M. 2006. The role of root exudates in rhizosphere interactions with plants and other organisms. Annu. Rev. Plant Biol. 57:233-266.

Bloemberg, G. V., and Lugtenberg, B. J. 2001. Molecular basis of plant growth promotion and biocontrol by rhizobacteria. Curr. Opin. Plant Biol. 4:343-350.

Cao, Y., Zhang, Z., Ling, N., Yuan, Y., Zheng, X., Shen, B., and Shen, Q. 2011. Bacillus subtilis SQR9 can control Fusarium wilt in cucumber by colonizing plant roots. Biol. Fertil. Soils 47:495-506.

Geels, F., and Schippers, B. 1983. Selection of antagonistic fluorescent Pseudomonas spp. and their root colonization and persistence following treatment of seed potatoes. J. Phytopathol. 108:193-206.

Germida, J. J., and Walley, F. L. 1996. Plant growth-promoting rhizobacteria alter rooting patterns and arbuscular mycorrhizal fungi colonization of field-grown spring wheat. Biol. Fertil. Soils 23:113-120.

Huang, S., Li, R., Zhang, Z., Li, L., Gu, X., Fan, W., Lucas, W. J., Wang, X., Xie, B., Ni, P., Ren, Y., Zhu, H., Li, J., Lin, K., Jin, W., Fei, Z., Li, G., Staub, J., Kilian, A., van der Vossen, E. A., Wu, Y., Guo, J., He, J., Jia, Z., Ren, Y., Tian, G., Lu, Y., Ruan, J., Qian, W., Wang, M., Huang, Q., Li, B., Xuan, Z., Cao, J., Asan, Wu, Z., Zhang, J., Cai, Q., Bai, Y., Zhao, B., Han, Y., Li, Y., Li, X., Wang, S., Shi, Q., Liu, S., Cho, W. K., Kim, J. Y., Xu, Y., Heller-Uszynska, K., Miao, H., Cheng, Z., Zhang, S., Wu, J., Yang, Y., Kang, H., Li, M., Liang, H., Ren, X., Shi, Z., Wen, M., Jian, M., Yang, H., Zhang, G., Yang, Z., Chen, R., Liu, S., Li, J., Ma, L., Liu, H., Zhou, Y., Zhao, J., Fang, X., Li, G., Fang, L., Li, Y., Liu, D., Zheng, H., Zhang, Y., Qin, N., Li, Z., Yang, G., Yang, S., Bolund, L., Kristiansen, K., Zheng, H., Li, S., Zhang, X., Yang, H., Wang, J., Sun, R., Zhang, B., Jiang, S., Wang, J., Du, Y., and Li, S. 2009. The genome of the cucumber, Cucumis sativus L. Nat. Genet. 41:1275-1281.

Hull, A. K., Vij, R., and Celenza, J. L. 2000. Arabidopsis cytochrome P450s that catalyze the first step of tryptophan-dependent indole-3-acetic acid biosynthesis. Proc. Natl. Acad. Sci. U.S.A. 97:2379-2384.
Idris, E. E., Iglesias, D. J., Talon, M., and Borriss, R. 2007. Tryptophandependent production of indole-3-acetic acid (IAA) affects level of plant growth promotion by Bacillus amyloliquefaciens FZB42. Mol. PlantMicrobe Interact 20:619-626.

Kamilova, F., Kravchenko, L. V., Shaposhnikov, A. I., Azarova, T., Makarova, N., and Lugtenberg, B. 2006a. Organic acids, sugars, and L-tryptophane in exudates of vegetables growing on stonewool and their effects on activities of rhizosphere bacteria. Mol. Plant-Microbe Interact 19:250-256.

Kamilova, F., Kravchenko, L. V., Shaposhnikov, A. I., Makarova, N., and Lugtenberg, B. 2006b. Effects of the tomato pathogen Fusarium oxysporum f. sp. radicis-lycopersici and of the biocontrol bacterium Pseudomonas fluorescens WCS365 on the composition of organic acids and sugars in tomato root exudate. Mol. Plant-Microbe Interact 19:1121-1126.

Livak, K. J., and Schmittgen, T. D. 2001. Analysis of relative gene expression data using real-time quantitative PCR and the $2^{(-\Delta \Delta C(T))}$ method. Methods 25:402-408.

Murashige, T., and Skoog, F. 1962. A revised medium for rapid growth and bio assays with tobacco tissue cultures. Physiologia. 15:473-497.

Shaharoona, B., Naveed, M., Arshad, M., and Zahir, Z. A. 2008. Fertilizerdependent efficiency of Pseudomonads for improving growth, yield, and nutrient use efficiency of wheat (Triticum aestivum L.). Appl. Microbiol. Biotechnol. 79:147-155.

Shao, J., Li, S., Zhang, N., Cui, X., Zhou, X., Zhang, G., Shen, Q., and Zhang, R. 2015a. Analysis and cloning of the synthetic pathway of the phytohormone indole-3-acetic acid in the plant-beneficial Bacillus amyloliquefaciens SQR9. Microb. Cell Fact. 14:130.

Shao, J., Xu, Z., Zhang, N., Shen, Q., and Zhang, R. 2015b. Contribution of indole-3-acetic acid in the plant growth promotion by the rhizospheric strain Bacillus amyloliquefaciens SQR9. Biol. Fertil. Soils 51:321-330.

Spaepen, S., Vanderleyden, J., and Remans, R. 2007. Indole-3-acetic acid in microbial and microorganism-plant signaling. FEMS Microbiol. Rev. 31:425-448.

Wan, H., Zhao, Z., Qian, C., Sui, Y., Malik, A. A., and Chen, J. 2010. Selection of appropriate reference genes for gene expression studies by quantitative real-time polymerase chain reaction in cucumber. Anal. Biochem. 399:257-261.

Wright, A. D., Sampson, M. B., Neuffer, M. G., Michalczuk, L., Slovin, J. P., and Cohen, J. D. 1991. Indole-3-acetic acid biosynthesis in the mutant maize orange pericarp, a tryptophan auxotroph. Science 254:998-1000.

Zhang, N., Wang, D., Liu, Y., Li, S., Shen, Q., and Zhang, R. 2014. Effects of different plant root exudates and their organic acid components on chemotaxis, biofilm formation and colonization by beneficial rhizosphereassociated bacterial strains. Plant Soil 374:689-700.

Zhou, T., Chen, D., Li, C., Sun, Q., Li, L., Liu, F., Shen, Q., and Shen, B. 2012. Isolation and characterization of Pseudomonas brassicacearum $\mathrm{J} 12$ as an antagonist against Ralstonia solanacearum and identification of its antimicrobial components. Microbiol. Res. 167:388-394.

\section{AUTHOR-RECOMMENDED INTERNET RESOURCE}

Cucurbit Genomics database: http://www.icugi.org/cgi-bin/ICuGI/index.cgi 